
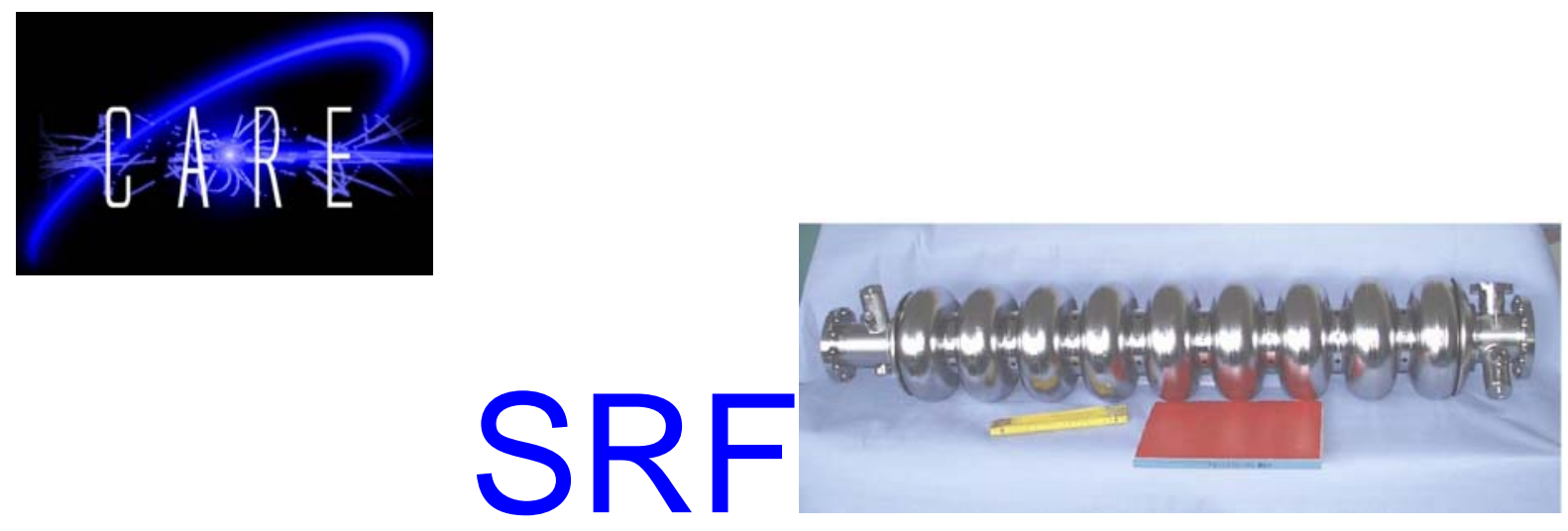

\title{
Deposition of Thin Metal Films by Means of Arc Discharges under Ultra-High Vacuum Conditions
}

Marek J. Sadowski, Pawel Strzyzewski , Robert Nietubyc

The Andrzej Soltan Institute for Nuclear Studies, Warsaw, Poland

\begin{abstract}
The paper reports on plasma technology of the production of thin metal layers by means of controlled arc discharges, initiated at ultra-high vacuum (UHV) conditions, i.e. at the background pressure $<10^{-8} \mathrm{~Pa}$, and carried out at the operating pressure $<10^{-5} \mathrm{~Pa}$. Different UHV arc facilities, which have been designed and operated at IPJ in Swierk, are described. The UHV arc facility equipped with a cylindrical cathode made of a pure niobium $(\mathrm{Nb})$ is applied for the deposition of superconducting Nb layers upon inner surfaces of RF accelerator cavities. The UHV arc device equipped with a truncated-cone cathode made of pure lead $(\mathrm{Pb})$ is used for the formation of photo-cathodes in RF electron guns. Filtering systems, which are used to reduce an amount of metal micro-droplets, are described. Characteristics of the deposited samples show that very pure and smooth metal films of the bulk-like crystalline structure can be produced by means of the described technique.
\end{abstract}

Contribution to the "IEEE EUROCON 2007", Warsaw (Poland), 9-12 September 2007

Work supported by the European Community-Research Infrastructure Activity under the FP6 "Structuring the European Research Area" programme (CARE, contract number RII3-CT2003-506395) 


\title{
Deposition of Thin Metal Films by Means of Arc Discharges under Ultra-High Vacuum Conditions
}

\author{
Marek J. Sadowski, Pawel Strzyzewski and Robert Nietubyc \\ The Andrzej Soltan Institute for Nuclear Studies (IPJ), \\ Department of Plasma Physics \& Technology (P-V) \\ 05-400 Otwock-Swierk n. Warsaw, Poland
}

\begin{abstract}
The paper reports on plasma technology of the production of thin metal layers by means of controlled arc discharges, initiated at ultra-high vacuum (UHV) conditions, i.e. at the background pressure $<10^{-8} \mathrm{~Pa}$, and carried out at the operating pressure $<10^{-5} \mathrm{~Pa}$. Different UHV arc facilities, which have been designed and operated at IPJ in Swierk, are described. The UHV arc facility equipped with a cylindrical cathode made of a pure niobium (Nb) is applied for the deposition of superconducting $\mathrm{Nb}$ layers upon inner surfaces of RF accelerator cavities. The UHV arc device equipped with a truncated-cone cathode made of pure lead $(\mathrm{Pb})$ is used for the formation of photocathodes in RF electron guns. Filtering systems, which are used to reduce an amount of metal micro-droplets, are described. Characteristics of the deposited samples show that very pure and smooth metal films of the bulk-like crystalline structure can be produced by means of the described technique.
\end{abstract}

\section{INTRODUCTION}

The deposition of thin metal films upon surfaces of various materials is of great importance for the material engineering. Such processes can be performed by means of different techniques, e.g., plasma vacuum deposition (PVD), magnetron sputtering, etc. [1]. In technology of modern RF-type accelerators one observe great interest in the application of superconducting materials. Since pure niobium $(\mathrm{Nb})$ is relatively expensive, the use of coppercavities coated with thin Nb-layers may reduce costs considerably. For this purpose the use was made of the magnetron sputtering technique [2], but quality of the deposited Nb-layers was not good enough. To improve adhesion and to reduce amount of impurities, a new technique based on cylindrical arc discharges under UHV conditions was proposed several years ago [3].

Another example is the formation of photo-cathodes needed for modern electron injectors in RF accelerators. Since the quantum efficiency of pure $\mathrm{Nb}$ is relatively low, it was proposed to use photo-cathodes made of lead $(\mathrm{Pb})$ [4]. To produce such photo-cathodes an UHV arc device with a planar (in fact a truncated cone) cathode made of pure $\mathrm{Pb}$ was applied [5].

The main aim of this paper was to describe different UHV facilities, which were designed and tested at IPJ in Swierk, Poland. Another aim was to show examples of the deposited metal $(\mathrm{Nb}$ or $\mathrm{Pb})$ layers and to present results of the investigation of their morphological and structural characteristics.

\section{EXPERIMENTAL FACILITIES}

\section{A. UHV arc facility with a cylindrical cathode}

In order to make possible the deposition of thin $\mathrm{Nb}$ layers upon internal surfaces of RF cavities, it was necessary to design a system based on the cylindrical configuration. The IPJ team, which collaborated with an Italian group at the Tor Vergata University in Rome and operated in a frame of the CARE EU project, designed and constructed the UHV arc facility equipped with a tubular cathode of $40 \mathrm{~mm}$ in diameter, which might be situated inside the cavity to be coated [6], as presented in Fig. 1.

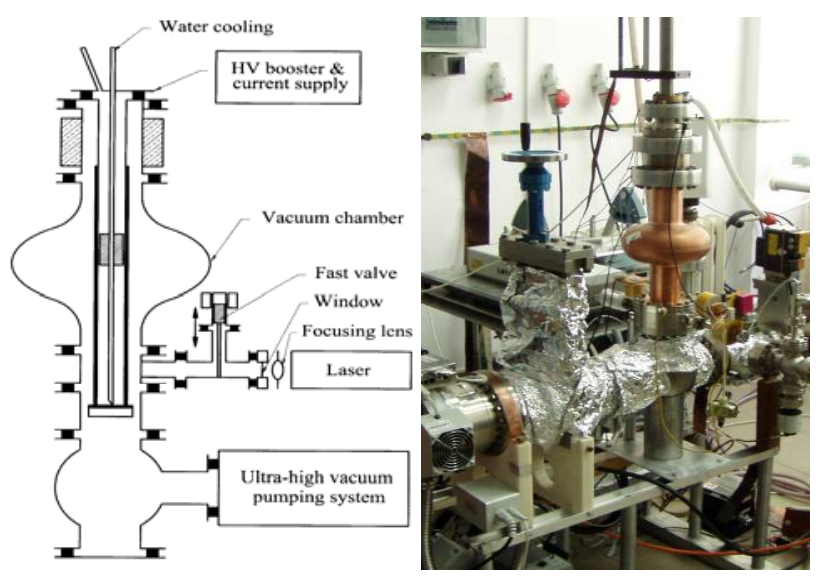

Fig.1. Schematic and view of UHV cylindrical-arc facility constructed at IPJ in Swierk.

Initially, in order to initiate arc discharges the use was made of an auxiliary electrode and high-voltahge trigger unit. Since such an initiation technique introduced some impurities from the trigger electrode and its insulator, it was decided to apply another initiation method based on a pulsed laser system [6] (see Fig. 1).

To make possible the uniform coating of the whole inner surface of the cavity, there is applied a strong permanent magnet driven inside the cathode in order to induce motion of the arc discharge along the cathode. 
To facilitate the deposition of $\mathrm{Nb}$ layers upon tests samples, e.g. upon sapphire or copper substrates, the described system was equipped with an exchangeable stain-less steel chamber of the shape similar to that of a TESLA-type cavity, but with diagnostic ports located in the horizontal plane.

The test chamber (or the original copper cavity) was initially pumped out to $10^{-6} \mathrm{~Pa}$ by means of an oil-free pumping system consisting of a two-stage piston pump and a turbomolecular pump. Particular attention was paid to the control of the composition of residual gases [7]. It was performed by means of a residual gas analyzer (RGA). Typical examples of mass-spectra, which correspond to the gas conditions before baking $\left(10^{-6} \mathrm{~Pa}\right)$ and after that $\left(10^{-8} \mathrm{~Pa}\right)$, are shown in Fig. 2 .
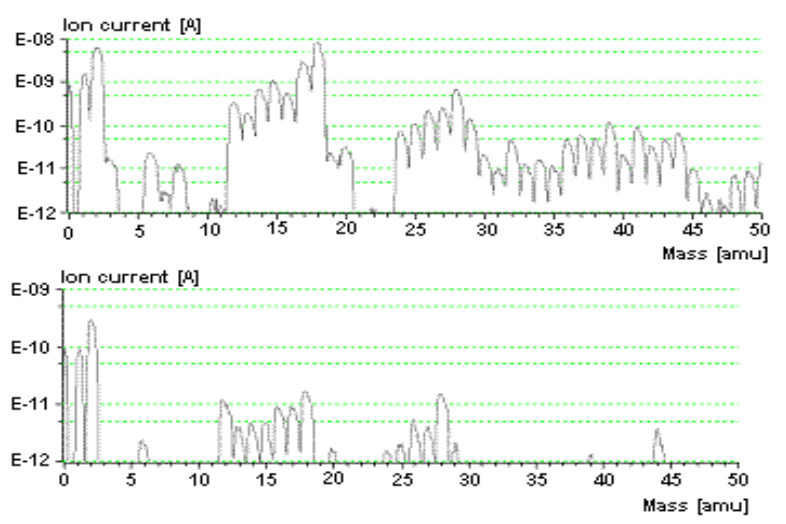

Fig. 2. Typical RGA mass-spectra recorded before and after 24-hour baking at $150{ }^{\circ} \mathrm{C}$.

The residual gas composition was also monitored before and during the arc operation, as shown in Fig. 3.

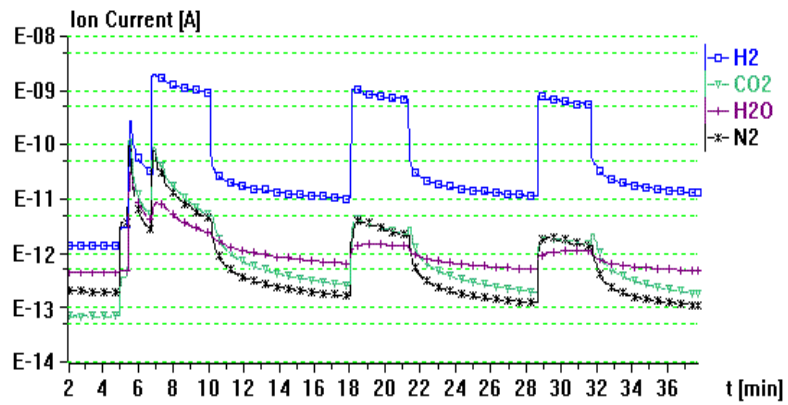

Fig.3. Partial pressures (in ion-current units) of residual gases during the arc triggering at the UHV conditions.

It can be seen that after the arc discharge initiation one can observe the out-gassing from the cathode. The pressure rises usually up to $10^{-5}-10^{-6} \mathrm{~Pa}$, but it remains almost stable during the deposition process. The gas pressure rise is caused mainly by hydrogen. At the UHV conditions the partial pressure of hydrogen is usually about 2-3 orders of magnitude higher than that of other gases. After 40-60 seconds after the arc initiation the partial pressures of out-gassed species began to fall. The higher partial pressure of the water vapor is probably caused by the formation of a fresh oxide-free $\mathrm{Nb}$ layer, which can absorb water remnants. When the arc is extinguished the hydrogen pressure drops about one order of magnitude, reaching its equilibrium level (in the presence of the $\mathrm{Nb}$ surface), and then it starts slowly to decrease due to pumping with the 200 1/s turbo-molecular pump.

\section{B UHV arc facility with a truncated-cone cathode}

The UHV devices with a truncated-cone (called also a planar) cathode were designed and constructed both at the Tor Vergata University in Rome and at IPJ in Swierk [3]. A schematic drawing of such a device is shown in Fig. 4.

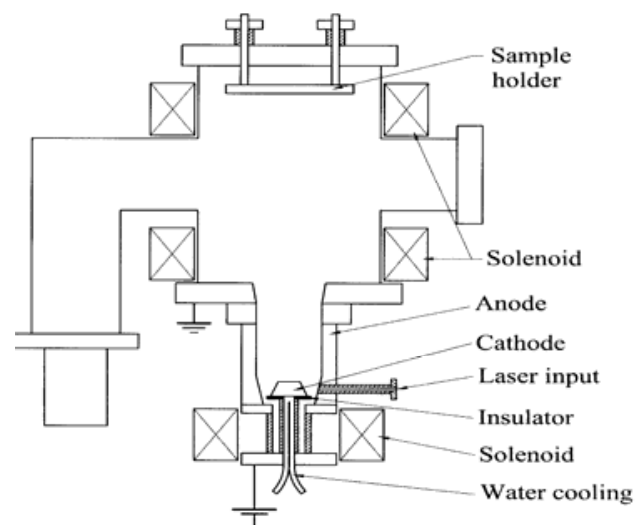

Fig. 4. Schematic of an UHV arc facility with a truncated-cone cathode and laser triggering.

The truncated-cone cathode (made of pure $\mathrm{Nb}$ or $\mathrm{Pb}$ ) is placed upon a water-cooled support inside a vacuum chamber made of a stainless-steel. To facilitate the motion of arc spots upon the cathode surface the use is made of an external magnetic coil. The experimental chamber is also surrounded with additional coils. Samples to be coated might be fixed upon an insulated holder which enables the polarization (bias) to be applied. The arc discharge is usually initiated with a pulsed laser beam focused upon the cathode surface.

The UHV arc sources with the truncated-cone cathode can easily be used for the deposition of metal films upon planar substrates or objects. They can also be applied for coating of the cavity inner surface, if they are placed at one or both necks of such a cavity.

It should also be noted that the described UHV arc devices are very convenient for studies of the different samples and on the optimization of the UHV arc operation.

\section{TEST DEPOSITIONS OF METAL FILMS}

\section{A. Film depositions in UHV arc facilities with a cylindrical cathode}

In order to check applicability of the UHV cylindrical arc device for the deposition of pure $\mathrm{Nb}$ layers upon the inner cavity surface the preliminary tests were carried out with a TESLA-type cavity model made of pure copper. 
After the optimization of the UHV conditions it was possible to perform the deposition process lasting about 2 minutes, at arc current equal to about $70 \mathrm{~A}$. The model cavity was coated with no filtering of micro-droplets, and after the deposition process it was cut along the symmetry axis to enable the inspection of uniformity of the deposited $\mathrm{Nb}$-layer to be performed. A picture of that model cavity is presented in Fig. 5.

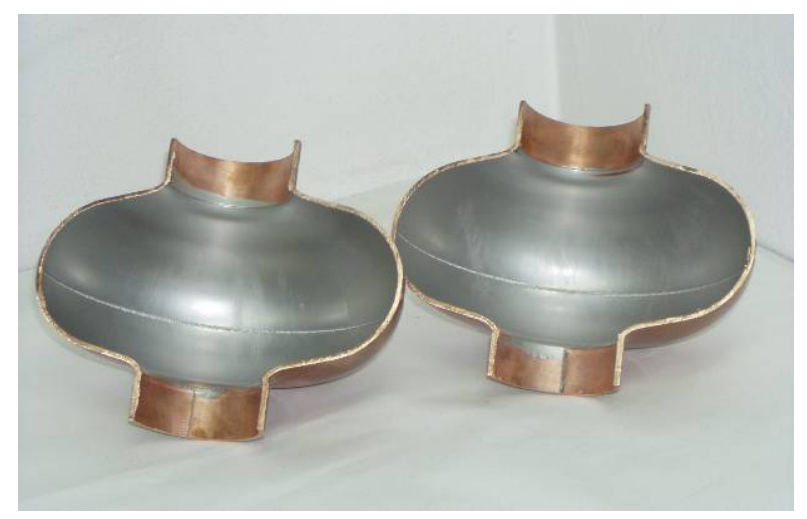

Fig.5. Photo of the model cavity, which demonstrates that the UHV arc facility with the cylindrical cathode can be applied for the deposition of a Nb-layer upon the whole inner surface.

To investigate the structure of the deposited $\mathrm{Nb}$ layer several samples were cut out of the coated model cavity and analyzed by means of the scattered electron microscopy (SEM) technique. An example of the SEM image is presented in Fig. 6.

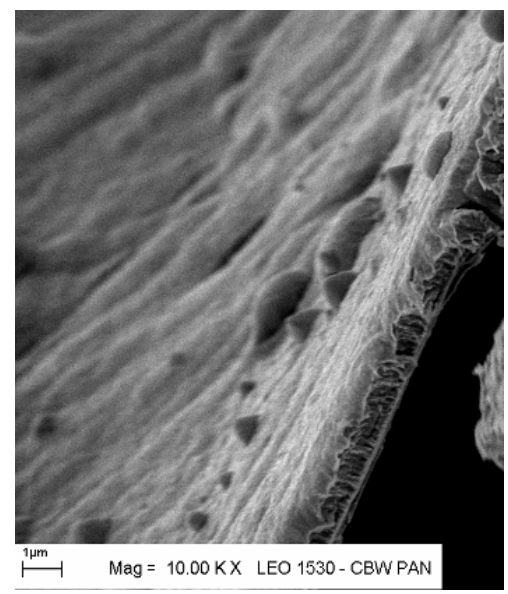

Fig.6. SEM picture of the cut-out sample, which shows the microstructure and thickness of the deposited Nb-layer.

From the SEM analysis one could easily conclude that the deposited $\mathrm{Nb}$ layer contains many micro-droplets, which should be eliminated if a very uniform superconducting film is required.

In order to determine the surface density of the deposited micro-droplets the use was made of the test stain-less steel chamber (described in Section IIA) with several diagnostic ports. Those ports were used to insert sapphire substrates, which after deposition were analyzed with an optical microscope and SEM. Some results are shown in Figs. 7 and 8.
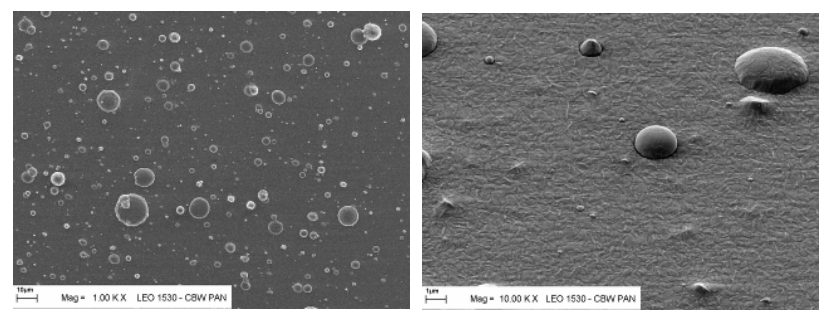

Fig.7. SEM pictures of a $\mathrm{Nb}$-coated sapphire sample, which show the micro-droplets population (on the left) as well as the structure of the $\mathrm{Nb}$-film and micro-droplets (on the right, with the larger magnification).

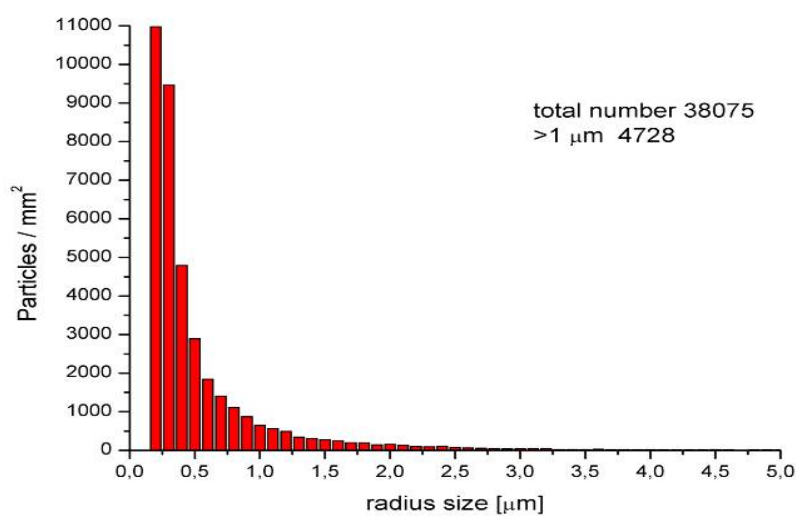

Fig.8. Surface density of the micro-droplets upon the sapphire sample coated with the $\mathrm{Nb}$-film, as measured with an optical microscope (courtesy of the Tor Vergata Uni team).

The test depositions described above showed that the problem of the elimination (or at least the reduction) of the deposited micro-droplets is of primary importance.

\section{B. Film depositions in UHV arc facilities with a truncated-cone cathode}

Test depositions of thin $\mathrm{Nb}$ films were also performed within UHV arc devices with a truncated-cone cathode, which were constructed at Tor Vergata University in Rome and IPJ in Swierk [3, 8-9]. In particular, very extensive studies of the optimization of the film deposition processes were carried out at the Tor Vergata laboratory. For this purpose the use was made of a special test chamber, consisting of two hemi-spherical parts made of stainless steel. Those parts were equipped with many holders, which enabled samples to be placed at different angles to the z-axis [10].

The arc discharge was initiated with a laser focused upon the surface of the truncated-cone cathode made of pure $\mathrm{Nb}$, and located below the test chamber. The transport of arc plasma through the whole system was investigated by means of ion collectors and samples placed inside the test chamber, particularly upon the upper hemi-spherical part. A schematic drawing and general view of the described experimental facility is presented in Fig. 9. 

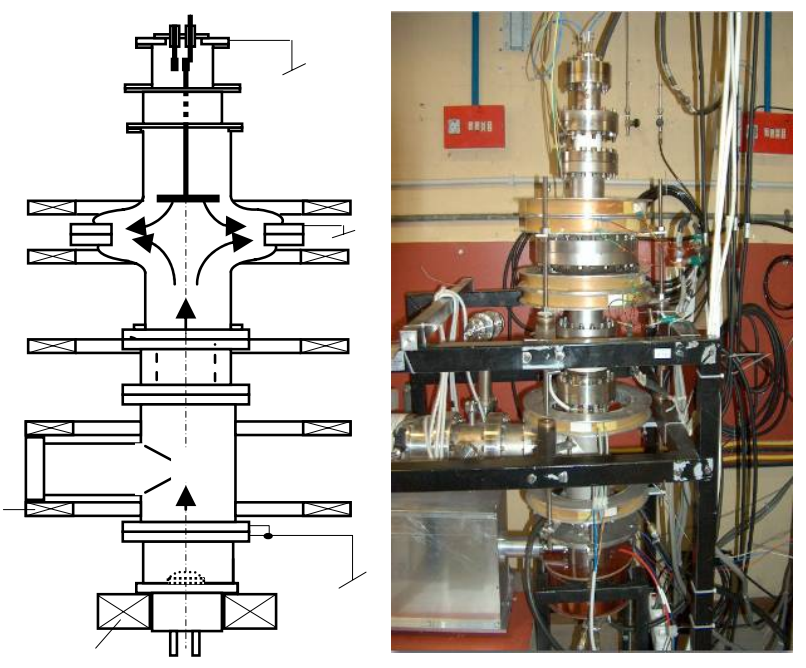

Fig.9. Schematic and view of the UHV arc facility with a truncatedcone cathode and two-part test chamber, which was constructed at Tor Vergata laboratory within a frame of the scientific collaboration [10].

Using the facility described above, detailed studies of morphology of the deposited $\mathrm{Nb}$ films were carried out by means of SEM. An example is shown in Fig. 10.

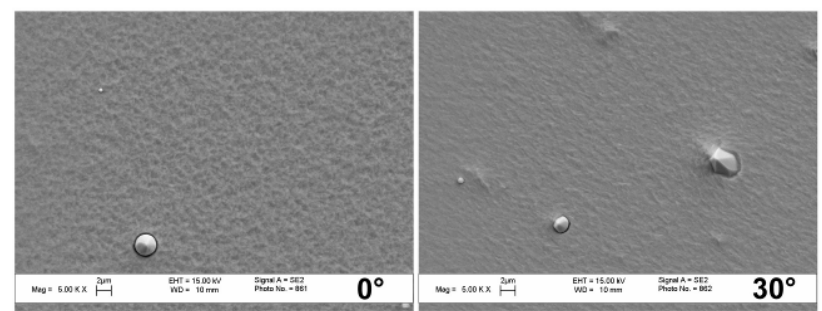

Fig.10. SEM pictures of the samples, which were placed at different angles to the symmetry axis of the system. One can easily notice some micro-droplets embedded into the deposited film or deposited upon its surface.

Detailed measurements of the most important parameters, and particularly of the RRR value (i.e. the ratio of the $\mathrm{Nb}$-layer resistance at a room temperature to that at a temperature of $10 \mathrm{~K}$ ), as well as the critical temperature, were carried out for many samples [10].

Using different values of the substrate bias and various coating times, the Tor Vergata team produced $\mathrm{Nb}$ layers of $1-3.5 \mu \mathrm{m}$ in thickness, with RRR values ranging from 25 to 50 (at the bias above $-40 \mathrm{~V}$ ).

It was proved that thin superconducting $\mathrm{Nb}$ films with properties of the bulk material can be deposited by means of the described UHV arc technology. The main difference in a comparison with the bulk $\mathrm{Nb}$ is the grain size which reaches about $300 \mathrm{~nm}$ (while in the bulk Nb it is about $200 \mathrm{~nm}$ ). It was also shown that $\mathrm{Nb}$ films have low impurity content (high RRR) and they show good RF properties [10].

Nevertheless, it appeared that the elimination of micro-droplets in UHV arc devices with a truncated-cone cathode is also of primary importance for further progress in the mastering of the described technology.

\section{FILTERS OF METAL MICRO-DROPLETS}

\section{A. Filters designed for UHV arc facilities with a cylindrical cathode}

In order to reduce the number of metal micro-droplets carried out by the arc discharge, different filtering systems of the cylindrical configuration were considered and designed. In the considered configuration relatively heavy micro-droplets are emitted from cathode spots mostly in radial directions and move along straight lines. Therefore, the simplest construction was based on Venetian-type blinds, which might be located around the cylindrical surface. Those blinds were arranged around the cathode, at the chosen radius and at appropriate angles and distances to eliminate the micro-droplets [9]. An example of such a filter is presented in Fig. 8.

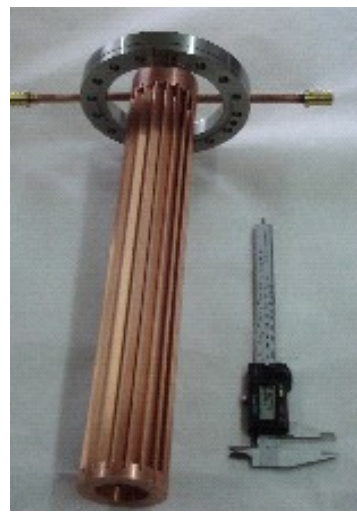

Fig.8. Picture of the Venetian-type filter designed for the UHV cylindrical arc facility.

To make possible the removal of heat deposited upon the filter surface, the supporting flanges (at the both filter ends) were water-cooled.

To facilitate the penetration of the arc discharge and metal ions through filter slits, it was proposed to use an active system with a magnetic field produced by currents, which might be put through neighbor blinds in opposite directions. To check the magnetic field configuration, appropriate computer modeling of the considered filter was performed, as shown in Fig. 9.

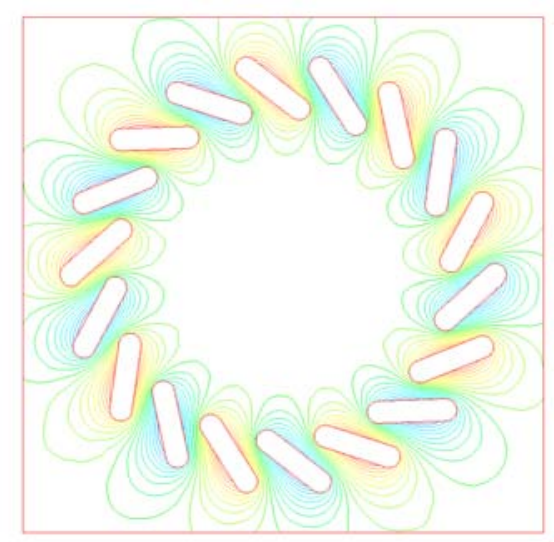

Fig.9. Distribution of magnetic field lines in the cross-section of the active cylindrical filter of the Venetian type. 
The modeling showed that within the considered configuration many magnetic field lines penetrate surfaces of the blinds, what can disturb the transport of metal ions. The construction of the Venetian-type filter with magnetizing currents would also be more complicated. Therefore, it was decided that preliminary tests of the micro-droplets filtering within the UHV cylindrical arc facility should be performed with the use of the simplest filter version without additional magnetic fields (see Fig.8).

To improve the distribution of magnetic field lines in the filter, it was proposed to use a concentric set of thin tubes distributed symmetrically upon the cylindrical surface [9]. Neighbor pairs of the tubes could draw magnetizing currents in the opposite directions and to create a better distribution of the magnetic field lines, as shown in Fig. 10.

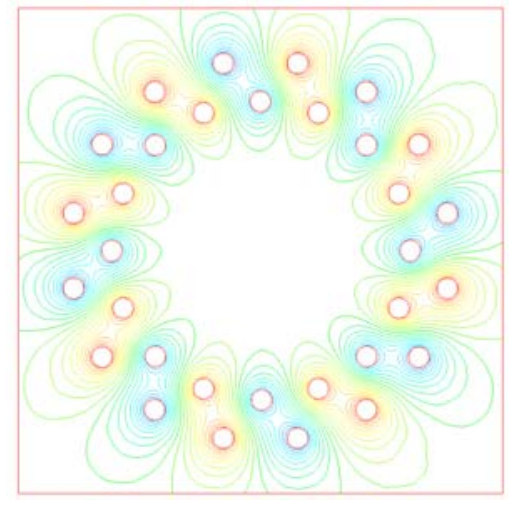

Fig.10. Distribution of magnetic field lines in the cross-section of the cylindrical filter of the multi-tube type.

It should be noted that the tubes can also be used for a water flow and to ensure more effective cooling of the filter. In spite of the fact that the construction of the described version of the cylindrical filter is relatively complicated, considering its advantages it was decided to manufacture a prototype, as shown in Fig. 11.

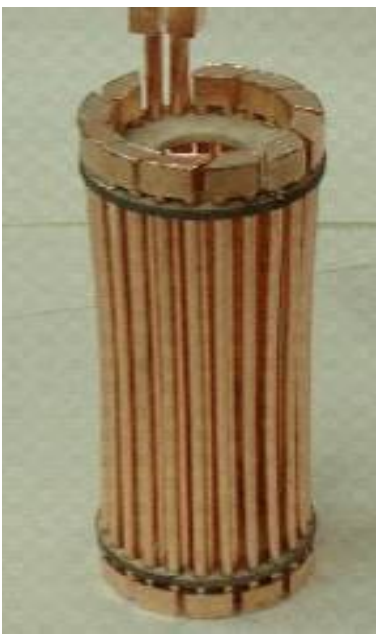

Fig.11. Picture of the prototype cylindrical filter consisting of thin tubes and appropriate connectors.
Experimental tests of the described filter within the UHV cylindrical arc facility are to be performed until the end of this year.

\section{B. Filters designed for UHV arc facilities with a truncated-cone cathode}

Other efforts were devoted to the design and construction of filters for the UHV arc facilities equipped with a truncated-cone cathode. In that case metal microdroplets are emitted mostly along the system symmetry axis and it is possible to apply a magnetic channel, which might deflect the arc discharge column while the microdroplets (moving mostly along straight lines) can be collected upon channel walls.

Different magnetic channels were considered and design. Particular attention was paid to $90^{\circ}$-knee filters of the Aksenov type, as well as to so-called T-type filters. Detailed computer modeling of the magnetic field lines in such filters was performed [8]. Some examples are presented in Figs. 12 and 13.

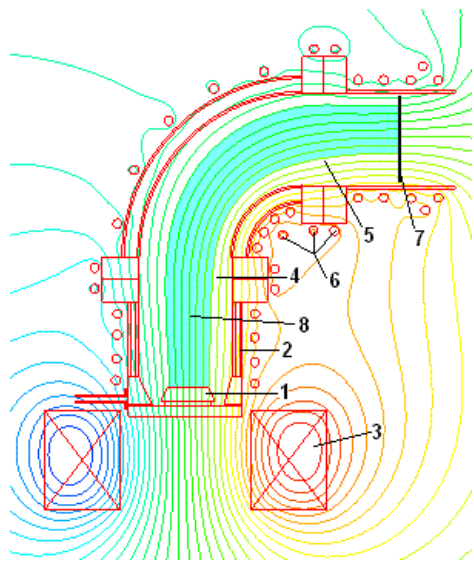

Fig.12. Modeling of the magnetic field lines distribution within the $90^{\circ}$-knee filter designed at IPJ in Swierk.

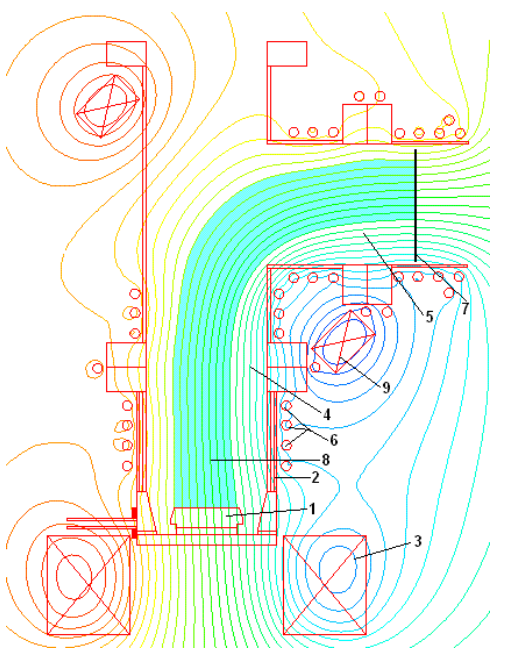

Fig.13. Modeling of the magnetic field lines distribution in a T-type filter. Notations: 1 - cathode, 2 - anode, 3 - focusing coil, 4 - filter inlet, 5 - filter exit, 6 - cable windings, 7 - ion collector, 8 - plasma stream, and 9 - correcting coil. 
Several versions of the described filters were manufactured and applied in different UHV arc facilities with truncated-cone cathodes, both at IPJ in Swierk and Tor Vergata University in Rome. A general view of one of those filters is shown in Fig. 14.

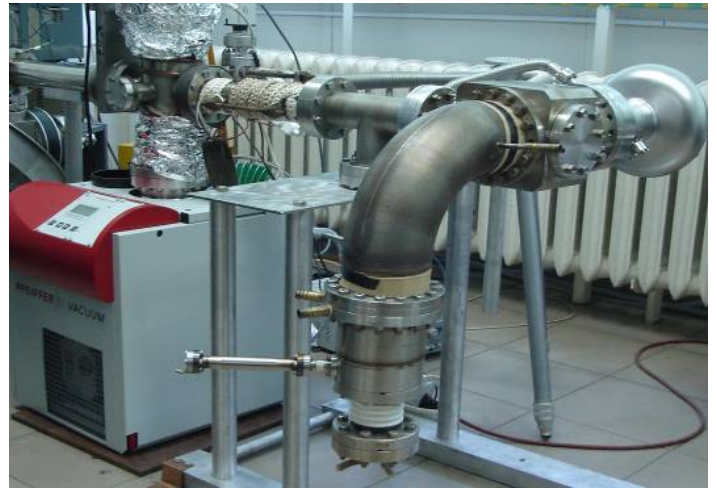

Fig.14. View of the UHV arc device with a truncated-cone cathode and the $90^{\circ}$-knee magnetic filter (without external windings), which was designed and constructed at IPJ in Swierk.

It should be noted that the described magnetic channel (shown in Figs. 12 and 14) had a double wall (to facilitate the water-cooling) and external windings, but it could also be equipped with additional correcting coils.

\section{RECENT EXPERIMENTAL RESULTS}

A. Recent depositions by means of the UHV arc facility with the cylindrical cathode made of pure $\mathrm{Nb}$

Operating within a frame of the CARE EU program, the IPJ team carried out numerous optimization tests of the UHV cylindrical arc facility (shown in Fig.1). Using the model vacuum chamber (described in Section IIA), several sapphire and copper substrates were coated with pure $\mathrm{Nb}$ layers under the optimized operational conditions.

The superconducting and structural properties of those samples were investigated. For the $\mathrm{Nb}$ films deposited without the use of the cylindrical filter, the RRR value was found to be in a range of $5-25$. That was probably connected with the relatively high population of micro-droplets observed on the deposited layers. A negative bias voltage, which was applied to the samples, reduced the surface density of micro-droplets considerably. It in turn resulted in the higher RRR values. Unfortunately, the biasing cannot be applied for the TESLA cavity coating within the UHV cylindrical arc facility, because the cavity must be kept at the ground potential.

To analyze the composition of the deposited $\mathrm{Nb}$-film the use was made of the scattered ion mass spectroscopy (SIMS) technique. The analysis performed with the use of Ar ions showed that the deposited film contained pure $\mathrm{Nb}$ and the concentrations of $\mathrm{NbO}$ and $\mathrm{NbH}$ were very low [11]. The SIMS profiles are presented in Fig. 15.

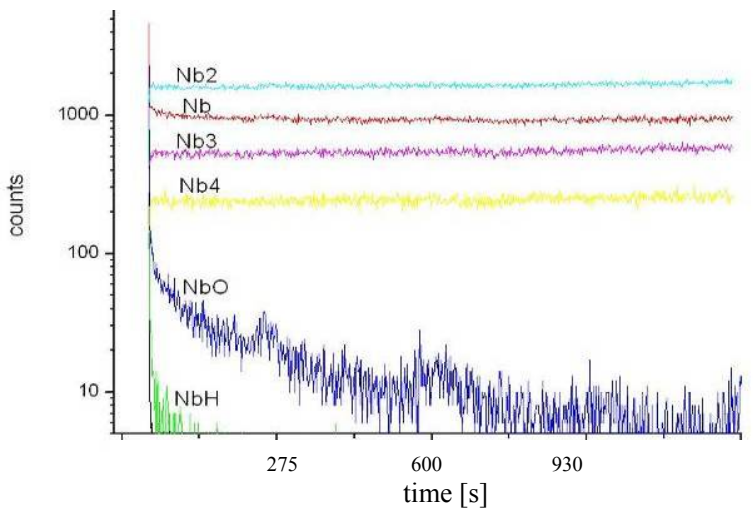

Fig.15. Results of the SIMS analysis of the Nb-film sample deposited within the UHV cylindrical arc facility.

Two samples of the $\mathrm{Nb}$ films, which were deposited upon sapphire (0001) substrates, have been analyzed by means of the X-ray diffraction method. Diffraction $\theta-2 \theta$ patterns have been measured using $\mathrm{Cu} \mathrm{K} \mathrm{K}_{\alpha}$ radiation $(\lambda=$ $1.54056 \AA$ ). The diffraction lines associated with films and substrates have been observed, as shown in Fig. 16.
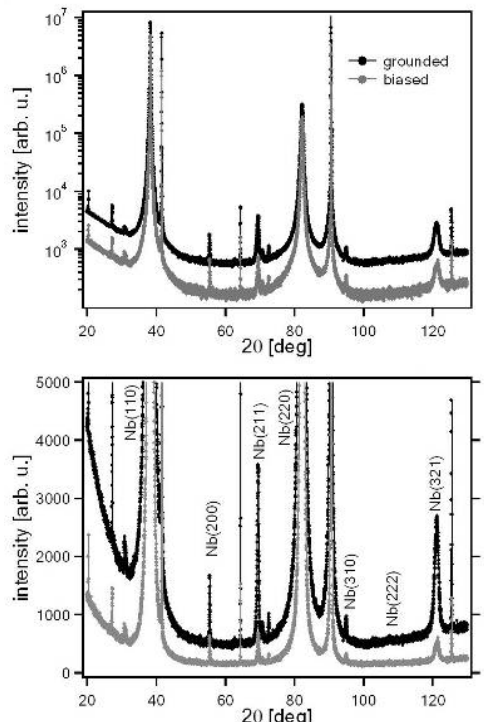

Fig.16. Results of the X-ray analysis of $\mathrm{Nb}$ layers deposited upon an unbiased sample (upper diagram) and that deposited with $-70 \mathrm{~V}$ bias (lower diagram).

An estimation of the out-of-plane lattice constants gave values of $3.3043 \pm 0.0017 \AA$ and $3.3043 \pm 0.0026 \AA$ for the grounded and biased sample, respectively. These values with their error bands are in agreement with the lattice constant of the perfect bcc $\mathrm{Nb}$ crystal, what says about quality of the deposited $\mathrm{Nb}$ layers.

Recently, particular efforts were oriented on the determination of filtering capabilities of cylindrical filters of the both types: passive (without magnetizing currents) and active (magnetic) ones [9]. The obtained $\mathrm{Nb}$ layers, which were coated with the use of the passive Venetianblind filter, showed that the surface density of microdroplets can considerably be reduced in a comparison with that observed for unfiltered depositions. Exemplary results of those measurements are presented in Fig. 17. 


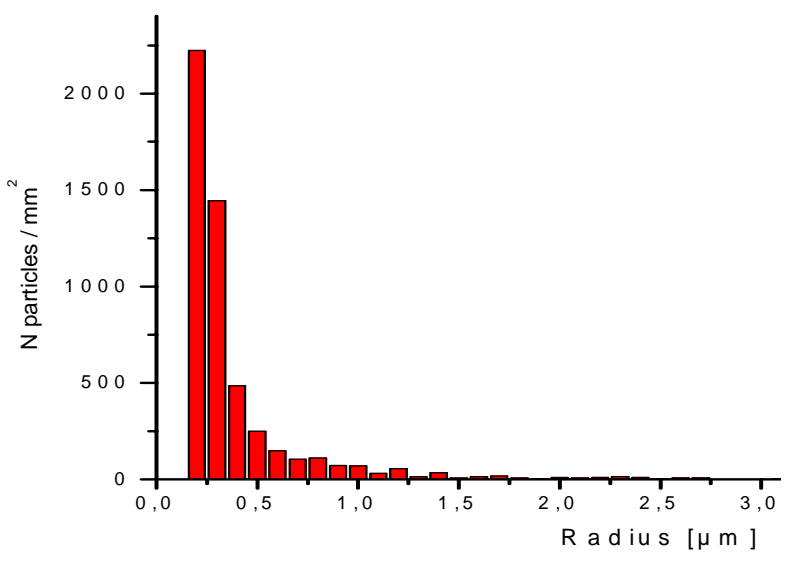

Fig.17. Surface population of micro-droplets as a function of their radii, which was measured upon the samples of pure $\mathrm{Nb}$ layers deposited with the use of the passive cylindrical filter of the Venetian type.

Unfortunately, the deposition of pure Nb-layers upon original copper cavities of the TESLA type could not be performed, because the manufacturing of these cavities has not been finished so far. Nevertheless, such tests are to be carried out this year.

\section{B. Recent depositions by means of the UHV arc facility with the truncated-cone cathode}

While the Tor Vergata team concentrated all efforts on the optimization of the deposition of pure $\mathrm{Nb}$ layers [10], the IPJ team continued research on the development of magnetic filters and the formation of photo-cathodes of pure $\mathrm{Pb}$ [8-9]. Nevertheless, some samples of the $\mathrm{Nb}$ films, which were deposited within the UHV arc facility equipped with a truncated-cone cathode made of pure $\mathrm{Nb}$, have also been investigated, as shown in Fig. 16.
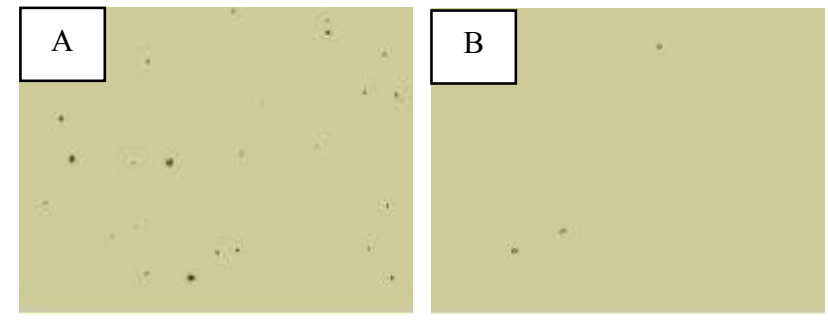

Fig.17. Photos of the samples coated with thin $\mathrm{Nb}$ films by means of UHV arc discharges, as obtained with different magnetic filters: (A) with the $90^{\circ}-$ knee filter, (B) with the T-type magnetic filter, which had higher efficiency of macro-droplets filtering [9].

Since the described tests showed that the T-type filter is better as regards the efficiency of the micro-droplets filtering, it has been decided that further experimental studies will be performed with the use of such a filter.

Recently, the experimental studies concerned mainly the $\mathrm{Pb}$ layers, which were deposited in the UHV arc facility equipped with a truncated-cone cathode made of pure $\mathrm{Pb}$. Several test deposition were performed and the obtained samples were investigated. Exemplary results of the SIMS analysis are shown in Fig. 18.

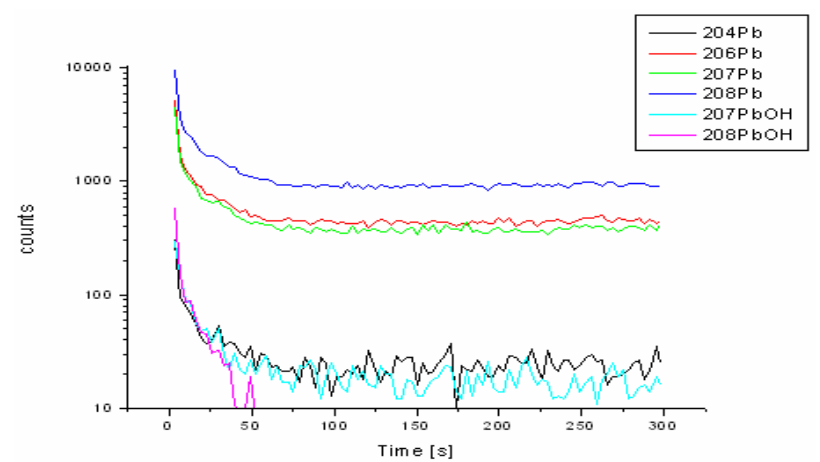

Fig.18. Results of the SIMS analysis of a Pb-film sample, which was deposited within the UHV arc facility equipped with a truncated-cone cathode[12].

In order to perform the surface quality inspection of the deposited $\mathrm{Pb}$ layers the use was made of SEM techniques. Using different magnifications it was possible to analyze the surface uniformity and to identify smallscale defects, as shown in Fig. 19.
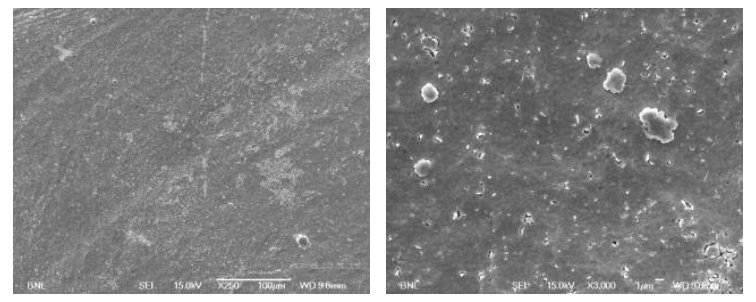

Fig. 19. SEM pictures of the $\mathrm{Pb}$ film deposited upon a copper substrate, which were taken at different magnifications.

The described analyses showed that the obtained $\mathrm{Pb}$ layers are dense and homogenous enough. A lack of micro-droplets upon the analyzed surfaces confirmed good efficiency of the plasma filtering. The quality of the $\mathrm{Pb}$ layers was also investigated for samples deposited upon sapphire substrates. In that case the roughness of the $\mathrm{Pb}$ layers was found to be of the order of few tenth of $\mathrm{nm}$.

To perform the deposition of a $\mathrm{Pb}$ film (photocathode) inside an RF Gun of the DESY type (i.e., a 1.3$\mathrm{GHz}$ cavity made of the bulk $\mathrm{Nb}$ ), some modifications of the existing facility (shown in Fig. 14) were necessary. The main problem was the plasma transport through the cavity channel of about $1 \mathrm{~m}$ in length and a relatively small diameter. Another problem was related with a bias of the RF Gun. In the described configuration the wall potential played an important role, making the plasma transport very difficult. In order to shield arc plasma against external electric fields, there was applied a special shielding tube, which was kept on ground potential [11]. Due to this shielding (of about $25 \mathrm{~cm}$ in length, and almost $5 \mathrm{~cm}$ in diameter) the plasma transport was relatively low. Measurements of the ion current were performed along the z-axis of the set-up. The deposition rate within the $1.3-\mathrm{GHz}$ cavity was about $0.2 \mathrm{~nm} / \mathrm{s}$.

Other tests were carried out with the deposition of $\mathrm{Pb}$ layers (photo-cathodes) upon plugs designed for an RF Gun of the JLab type. Since those plugs were relatively 
small, they could be placed upon a special holder located 6 . inside the vacuum chamber, near the magnetic filter outlet. Therefore, in a comparison with the previous case, the deposition rate was 7-8 times higher [11]. It should be noted that the holder, similarly as the DESY RF Gun described above, were insulated from walls of the vacuum chamber and a negative bias of $20-100 \mathrm{~V}$ could be applied, both in the DC and kHz-pulse regimes.

In order to compare the vacuum-arc deposited $\mathrm{Pb}$ photo-cathodes with those obtained with other techniques, a special experimental setup for Quantum Efficiency (QE) measurements was prepared at the BNL in USA [12]. The investigated samples were illuminated with $\mathrm{KrF}$ Excimer, ArF Excimer, and Nd:YAG (4th harmonic) laser pulses. The QE data, as obtained at BNL, for different $\mathrm{Pb}$ photocathodes, are presented in Fig. 20.

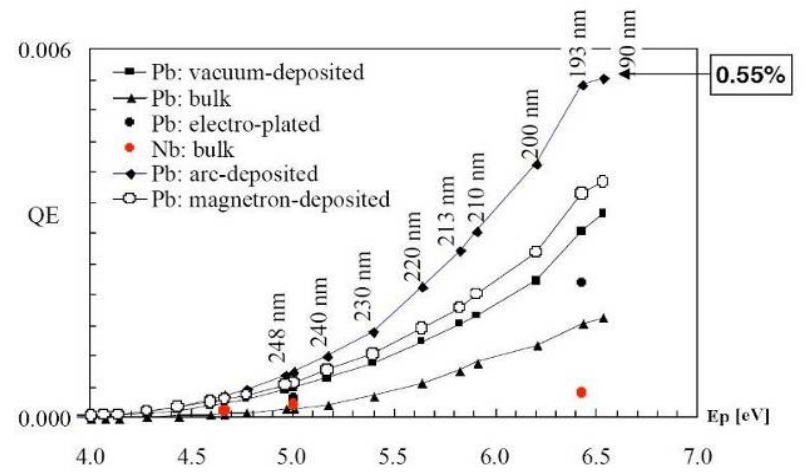

Fig.20. Results of QE measurements of different photo-cathodes [12].

Those studies showed that the best QE values have been obtained with the $\mathrm{Pb}$ photo-cathodes deposited by means of the UHV arc discharges. It opens a new road for applications of this technology.

\section{SUMMARY AND CONCLUSIONS}

The most important results of the studies reported above can be summarized as follows:

1. New experimental facilities based on arc discharges under ultra-high vacuum (UHV) have been developed and investigated.

2. It has been shown that the deposition processes, which are realized by means of UHV arc discharges, can guarantee a very small amount of unwanted impurities within the deposited films.

3. To eliminate metal micro-droplets different systems of passive and active (i.e. magnetic) filters have been designed and tested.

4. High quality of the UHV arc-deposited films and thicker layers has been confirmed by detailed analyses performed with different techniques (SEM, SIMS, GD-OES, etc.) as well as by measurements of their superconducting properties.

5. Test coatings of the inner surface of single copper RF cavity with a pure $\mathrm{Nb}$ layer have already been performed successfully, but they must be carried out for the original TESLA-type cavities.
The depositions of pure $\mathrm{Pb}$ spots inside different models of RF electron guns, as designed for modern linear accelerators, have been performed successfully and the produced $\mathrm{Pb}$ photo-cathodes show very good characteristics.

In conclusion it can be stated that the described UHV arc technology requires still some improvements, particularly in the micro-droplets filtering, but it evidently opens a new road to many scientific and technological applications. This technology might be used when dense, high-quality and very pure metal films are needed, e.g., in micro-electronics, medicine, nanotechnology etc.

\section{ACKNOWLEDGMENT}

We acknowledge a support of the EC - Research Infrastructure Activity under the FP6 "Structuring the European Research Area" program (CARE, contract number RII3-CT-2003-506395). We would also like to thank Dr W. Paszkowicz of the Institute of Physics, P.A.S., Warsaw, for the X-ray analysis of the samples.

\section{REFERENCES}

[1] S. Anders, "From plasma immersion ion implantation to deposition: a historical perspective on principles and trends", Surf. Coat. Tech. 156 (2002) 3.

[2] C. Benvenuti, S. Calatroni, I..E. Campisi, et al., "Properties of copper cavities coated with niobium using different discharge gases", in Proc. $8^{\text {th }}$ Workshop on RF Superconductivity, Abano Terme PD, Italy, October 1997, Vol.4, pp. 1057+1064.

[3] J. Langner, L. Catani, M. Cirillo, et al., "Formation of thin superconducting films by means of ultra-high vacuum arc", Czech. J. Phys. Vol. 52, Suppl. D (2002) D829-D835.

[4] J. Sekutowicz, J. Iversen, G. Kreps, et al., "Nb-Pb superconducting RF gun", TESLA-FEL Report 2005-09, DESY, Hamburg, Germany, December 2005.

[5] P. Strzyzewski, J. Langner, M.J. Sadowski, et al., "Deposition of lead thin films used as photo-cathodes by means of cathodic arc under UHV conditions", in Proc. of EPAC 2006, Edinburgh, Scotland, June 2006, pp. 3205-3207.

[6] J. Langner, M.J. Sadowski, K. Czaus, et al., "Research on initiation of ultra-high vacuum arc discharges applied for deposition of thin superconducting layers", in Proc. Intern. Conf. PLASMA-2003, Warsaw, Poland, September 2003, p. O-9.3.

[7] P. Strzyzewski L. Catani, A. Cianchi, et al., "Behaviour of gas conditions during vacuum arc discharges used for deposition of thin films", in Proc. Intern. Conf. PLASMA-2005, Opole, Poland, Sept. 2005; AIP CP Vol. 812 (2006) 485-488.

[8] P. Strzyzewski, J. Langner, R. Mirowski, et al., "Magnetic filters in UHV arc discharges: constructions, field modeling and tests of efficiency", Phys. Scr. T123 (2006) 135-139.

[9] M.J. Sadowski, J. Langner, R. Mirowski, et al., "Progress in research on deposition of thin superconducting films by means of ultra high vacuum arc discharges", in Proc. Intern. Workshop on Thin Films, Padua-Legnaro, Italy, October 2006, http://master.lnl.infn.it/thinfilms.

[10] L. Catani, A. Cianchi, J. Lorkiewicz, et al., "The UHV cathodic arc: results on $\mathrm{Nb}$ films and plasma transport for cavity coating", in Proc. Intern. Workshop on Thin Films, Padua-Legnaro, Italy, October 2006, http://master.lnl.infn.it/thinfilms.

[11] P. Strzyzewski, J. Langner, M.J. Sadowski, et al., "Deposition of pure lead photo-cathodes by means of UHV cathodic arc", in Proc. Intern. Workshop on Thin Films, Padua-Legnaro, Italy, October 2006, http://master.lnl.infn.it/thinfilms.

[12] J. Sekutowicz, J. Iversen, J. Klinke, et al.,"Nb-Pb superconducting RF gun"; in Proc. of EPAC 2006, Edinburgh, Scotland, June 2006, pp. 3489-3491. 